\title{
CHECKLIST PARA PASSAGEM DE PLANTÃO DE PACIENTES EM PÓS-OPERATÓRIO IMEDIATO NA ADMISSÃO EM TERAPIA INTENSIVA
}

Sabrina Guterres da Silva ${ }^{1}$, Eliane Regina Pereira do Nascimento ${ }^{2}$, Patricia Madalena Vieira Hermida ${ }^{3}$ Adnairdes Cabral de Sena ${ }^{4}$, Taise Costa Ribeiro Klein 5 , Fabiana Minati de Pinho ${ }^{6}$

Objetivo: conhecer a percepção dos profissionais de enfermagem sobre a passagem de plantão e construir um checklist para passagem de plantão de pacientes em pós-operatório imediato admitidos na Terapia Intensiva. Metodologia: estudo qualitativo, exploratório e descritivo, realizado em hospital público de Santa Catarina, com entrevista semiestruturada a 55 profissionais de Enfermagem da Terapia Intensiva e Centro Cirúrgico. Na análise, se utilizou o Discurso do Sujeito Coletivo. Resultados: os relatos originaram as ideias centrais: "Relação entre a passagem de plantão e a segurança do paciente" e "Informações necessárias para sistematização da passagem de plantão". Conclusões: a passagem de plantão é percebida como essencial ao planejamento do cuidado seguro e o checklist contemplou informações acerca da identificação do paciente, das intercorrências no Centro Cirúrgico e dispositivos invasivos. A objetividade do checklist poderá facilitar sua implementação.

Descritores: Continuidade da assistência ao paciente, Terapia intensiva, Enfermagem de centro cirúrgico, Lista de checagem.

\section{CHECKLIST FOR NURSING SHIFT CHANGES OF PATIENTS IN IMMEDIATE POST SURGERY TO THE ADMISSION IN INTENSIVE CARE}

Aim: to know nursing professionals' perception of the shift change and develop a checklist for shift changes of patients in immediate post-surgery admitted to the Intensive Care. Methodology: qualitative, exploratory and descriptive research conducted in a public hospital of Santa Catarina, with a semi-structured interview to 55 nursing professionals of the Intensive care and Surgical Center. Results: the Collective Subject Discourse was used in the analysis. The reports originated the following central ideas: "Relationship between the shift change and patient safety" and "Necessary information for the shift change systematization." Conclusions: the nursing shift change is perceived as essential for a safe care planning, and the checklist included information about patient's identification, intercurrences at the Surgical Center and invasive devices. The checklist objectivity could facilitate its implementation.

Descriptors: Continuidad de la asistencia al paciente, Terapia intensiva, Enfermería del centro quirúrgico, Lista de comprobación.

\section{CHECKLIST PARA EL PASE DE GUARDIA DE PACIENTES EN POS-OPERATORIO INMEDIATO EN LA ADMISIÓN EN TERAPIA INTENSIVA}

Objetivo: conocer la percepción de los profesionales de enfermería sobre el pase de la guardia y construir una checklist para los pacientes en pos-operatorio inmediato admitidos en Terapia Intensiva. Metodología: estudio cualitativo, exploratorio y descriptivo realizado en un hospital público de Santa Catarina, con una entrevista semiestructurada de 55 profesionales de enfermería, de Terapia Intensiva y del Centro Quirúrgico. Se utilizó, en el análisis, el Discurso del Sujeto Colectivo. Resultados: los relatos originaron las siguientes ideas centrales: "Relación entre el pase de guardia y la seguridad del paciente" e "Informaciones necesarias para la sistematización del pase de guardia". Conclusiones: el pase de guardia es considerado como esencial para el planeamiento del cuidado seguro y el checklist contempló informaciones acerca de la identificación del paciente, de los acontecimientos en el Centro Quirúrgico y los dispositivos invasivos. La objetividad del checklist podrá facilitar su implementación.

Descriptores: Continuidad de la asistencia al paciente, Terapia intensiva, Enfermería del centro quirúrgico, Lista de comprobación.

${ }^{1}$ Enfermeira. Doutoranda do Programa de Pós-Graduação em Enfermagem da Universidade Federal de Santa Catarina (UFSC). Florianópolis - SC - Brasil. ${ }^{2}$ Enfermeira. Doutora em Enfermagem. Professora do Departamento de Enfermagem e do Programa de Pós-Graduação em Enfermagem da UFSC. Florianópolis - SC - Brasil. pongopam@terra.com.br.

${ }^{3}$ Enfermeira. Doutoranda do Programa de Pós-Graduação em Enfermagem da UFSC. Florianópolis - SC - Brasil.

${ }^{4}$ Enfermeira. Mestre em Enfermagem. Coordenadora de Enfermagem Cirúrgica do Hospital Universitário da UFSC. Florianópolis - SC - Brasil.

${ }^{5}$ Enfermeira. Mestre em Enfermagem. Enfermeira do Serviço de Controle da Infecção Hospitalar do Hospital Universitário da UFSC. Florianópolis - SC - Brasil. ${ }^{6}$ Enfermeira. Mestre em Enfermagem. Enfermeira da Unidade da Terapia Intensiva do Hospital Universitário da UFSC. Florianópolis - SC - Brasil. 


\section{INTRODUÇÃO}

A passagem de plantão é momento em que ocorre a troca de informações entre os profissionais e são abordadas questões referentes ao estado dos pacientes, assistência prestada, intercorrências e assuntos de interesse institucional( ${ }^{(1)}$. As informações transmitidas devem ser claras e precisas, pois falhas comunicativas podem representar prejuizos na assistência, inclusive com danos à saúde dos pacientes ${ }^{(2)}$.

A passagem de plantão é indispensável na transferência de pacientes entre setores e/ou instituições de saúde, portanto, fundamental que seja sistematizada e efetiva. Recomendações relativas à transferência interna e passagem de plantão dos pacientes têm sido contempladas em diferentes estudos ${ }^{(3,4)}$, na busca de melhorias à segurança do paciente no contexto hospitalar.

Situação bastante peculiar da passagem de plantão entre setores, é quando pacientes em Pós-Operatório Imediato (POI) são admitidos em Unidades de Terapia intensiva (UTI). Nesses casos, geralmente ela ocorre na presença do paciente, durante a sua transferência para o leito, monitorização, entre outras atividades realizadas concomitantemente. Nessa ocasião, há risco para desatenção principalmente devido aos ruídos, que podem gerar falhas e comprometer a assistência. Logo, a sistematização da passagem de plantão mediante a utilização de um impresso pode contribuir para que as informações do intraoperatório sejam recebidas de maneira organizada e correta, sem perdas ocasionais por comunicação verbal em ambiente ruidoso, como da UTI(5).

Esse estudo teve como objetivo conhecer a percepção dos profissionais de Enfermagem sobre a passagem de plantão e construir, a partir de seus discursos, um checklist para passagem de plantão de pacientes em POI admitidos na UTI.

Os checklists foram adaptados para o contexto da saúde e, além de auxiliar a tomada de decisão, possibilitam a segurança do paciente, reduzem riscos e custos. Sua utilização não visa apenas produzir registros, mas favorecer o diálogo entre a equipe, a fim de garantir a execução das tarefas e que todos façam o necessário para obter o melhor resultado(6)

\section{METODOLOGIA}

Estudo qualitativo, exploratório e descritivo, realizado em UTI e Centro Cirúrgico (CC) de um hospital público de Santa Catarina. Os dados foram coletados deagosto de 2010 a maio de 2011, com entrevista semiestruturada sobre a percepção dos participantes da passagem de plantão e sugestões de informações importantes para constar no checklist de passagem de plantão do CC para UTI. As entrevistas foram individuais, em ambiente privativo, gravadas e transcritas. Os critérios de inclusão eram: ser da equipe de Enfermagem; e atuar no CC ou na UTI. Os critérios de exclusão foram: profissionais em licença ou férias no período de coleta dos dados.

Os dados foram organizados e analisados em duas etapas. Na primeira, empregou-se o método do Discurso do Sujeito Coletivo (DSC), com três figuras metodológicas: expressões-chaves (ECHs) - trechos que revelam a essência do conteúdo do discurso e que devem ser destacados; Idéias Centrais (ICs) - expressões linguísticas que nomeiam sinteticamente o(s) sentido(s) presente(s) em cada uma das respostas; e, DSC - discurso-sintese, elaborado com ECHs de mesmo sentido e que têm a mesma IC, construido na primeira pessoa do singular como se fosse o discurso de somente um sujeito ${ }^{(7)}$.

$\mathrm{Na}$ segunda etapa, se elaborou o Checklist com base nas respostas dos participantes, algumas apresentadas nos DSCs acerca das informações que deveriam contemplar esse instrumento. Essas informações foram agrupadas conforme os dois momentos da passagem de plantão: do CC para a UTI - antes do encaminhamento do paciente, e na admissão na UTI. Para o primeiro momento, cuja comunicação ocorre por telefone, de enfermeiro para enfermeiro e com o paciente ainda no CC, foram agrupadas as informações de identificação do paciente, tipo de cirurgia, dentre outros. Para o segundo momento, se agrupou os dados vinculados às intercorrências no transopoeratório, às condições neurológicas e circulatórias, e dispositivos.

Os participantes foram identificados com as letras "E" (enfermeiro) e "T" (técnico de enfermagem), seguidas dos números correspondentes à sequência das entrevistas e das abreviaturas CC e UTI. A pesquisa foi aprovada pelo Comitê de Ética em Pesquisa da Universidade Federal de Santa Catarina (Processo 859/FR 350112) CAAE: 0145.0.242.000-10, e conforme Resolução 196/96, do Conselho Nacional de Saúde.

\section{RESULTADOS}

Dos 55 participantes do estudo, 43 eram do sexo feminino, 10 enfermeiros, sendo 02 do $\mathrm{CC}$. Os demais 45 eram técnicos de enfermagem, dos quais 30 da UTI. Emergiram dos relatos duas IC: Relação entre a passagem de plantão e a segurança do paciente que teve a expressão de 15 profissionais; e, Informações necessárias para sistematização da passagem de plantão - que envolveu oito profissionais. As ICs originaram os DSC 1 e DSC 2.

\section{IC: Relação entre a passagem de plantão e a segurança do paciente.}

DSC 1: A passagem de plantão é muito importante, pois nesse momento posso conhecer um pouco da história do paciente, saber que tipo de procedimento foi realizado e se houve intercorrências no transoperatório. Na UTI, deve-se saber tudo o que ocorreu com o paciente no CC para dar continuidade na assistência. Além disso, a equipe precisa estar informada para preparar o leito, materiais, equipamentos antecipadamente, para oferecer um atendimento de qualidade. Quanto mais informações melhor, pois o cuidado se torna personalizado, garantindo a segurança do paciente. (TIUTI, T5UTI, T1OUTI, T13UTI, T15UTI, T16UTI, T19UTI, T2OUTI, T21UTI, T23UTI, T2CC, T3CC, T8CC, Tl1CC, T13CC)

Este DSC revela que os profissionais consideram essencial a passagem de plantão entre a equipe de enfermagem do CC e da UTI, tratando-se de pacientes em POI. Ainda no DSC 1, os profissionais sugerem os registros/informações prioritários à sistematização da passagem de plantão nessas situações de cuidado. 
IC: Informações necessárias para sistematização da passagem de plantão.

DSC 2: Na passagem de plantão do CC para a UTI é importante que esteja incluso no checklist: nome do paciente, idade, estado de saúde, história de doenças prévias, tipo de cirurgia, tipo de anestesia, nivel de consciência, se houve intercorrências como sangramento e se precisou de hemotransfusão. É necessário saber se virá com drenos e sondagens e quais suas localizações e drenagens. Além disso, é importante ter ciência do tipo de ventilação (espontânea ou mecânica), o acesso venoso (profundo ou periférico), estado hemodinâmico, se está com PAM e/ou PVC, se virá com drogas de infusão contínua, tudo para que se possa organizar previamente os materiais e equipamentos para receber esse paciente na UTI. (EICC, E4UTI, E6UTI, T2UTI, T9UTI, T19UTI, T11CC, T13CC)

As informações relatadas no DSC 1 e mais detalhadas no DSC 2 subsidiaram a elaboração do checklist disponibilizado no Quadro 1

Quadro 1 - Checklist para passagem de plantão da enfermagem do CC para UTI adulta. Florianópolis,

SC, Brasil, 2012

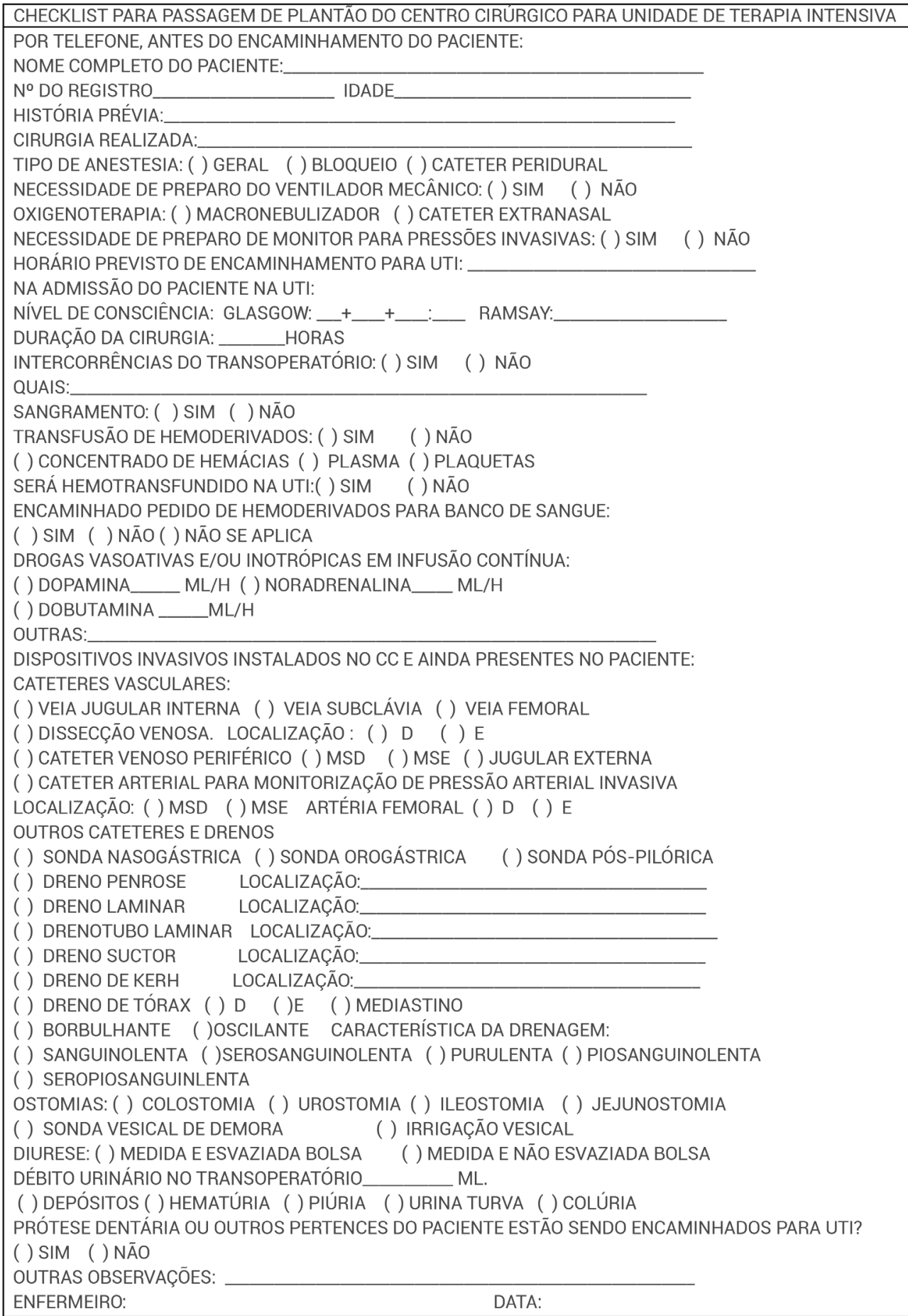




\section{DISCUSSÃO}

No DSC 1, os profissionais definem a passagem de plantão como umveículo de cuidado que promovea segurança do paciente, entretanto, para alcançá-la de fato algumas competências são necessárias, sendo a comunicação a mais evidente.

Diferentes formas de comunicação podem ser adotadas, contudo, a verbal e a escrita são as mais comuns, destacandose a primeira. $\mathrm{Na}$ assistência de enfermagem, a comunicação verbal, frequentemente empregada entre o CC e a UTI, ocorre por telefone. Interferências, como ruídos, outros profissionais chamando atenção e conversas paralelas podem causar falhas na transmissão da informação(5). Pesquisas apontam que falhas na comunicação são fatores que mais contribuem para erros e danos à saúde de pacientes ${ }^{(8)}$. Há uma cultura de segurança desfavorável relacionada às comunicações e atividades externas ao setor de trabalho, bem como deficiências na passagem de plantão(9, 10)

Estudo aponta que, apesar da fraca evidência disponível na literatura, a passagem de plantão padronizada entre setores de uma mesma instituição tem um impacto positivo em muitos processos e resultados, como: melhora da comunicação; reduz as complicações; elimina os atrasos na admissão do paciente; melhora a compreensão das suas condições de saúde; proporciona uso mais efetivo do tempo e diminui a duração da passagem de plantão(11).

Há instituições onde inexiste a comunicação entre o CC e o enfermeiro da UTI, recebendo o paciente sem informações do intraoperatório. A busca das informações ocorre no prontuário, o que demanda tempo e atrasa o planejamento do cuidado. Assim, o enfermeiro do CC deve transferir ao enfermeiro da unidade de destino as informações fidedignas sobre o paciente no intraoperatório, contribuindo com o planejamento da assistência no $\mathrm{POI}^{(3,5)}$

Estudo sobre um processo de transferência aprimorado com o uso de um checklist, empregado na transferência de crianças para a UTI após cirurgia cardíaca, constatou, depois de cinco anos da aplicação inicial desse processo aprimorado, redução substancial nas omissões de informação verbal e erros técnicos. Concluiu-se que processos de transferência padronizados podem ser uma estratégia sustentável para melhorar a segurança do paciente após cirurgia cardíaca pediátrica ${ }^{(12)}$.

No Brasil, instrumento elaborado para sistematizar a passagem de plantão entre duas unidades críticas melhorou a comunicação e promoveu a continuidade do cuidado. A comunicação escrita demanda menor desgaste dos enfermeiros dessas unidades, que não precisam interromper suas atividades para trocar informações por telefone, por exemplo, o que pode evitar interferências nesse processo comunicativo(5).

Nessa perspectiva, no DSC 2 deste estudo, a equipe revela informações fundamentais para auxiliar a comunicação na passagem de plantão e o planejamento do cuidado no POI na UTI, as quais são prioritárias na constituição do checklist.

Os participantes deste estudo consideram que dados como nome e idade são essenciais para o planejamento da assistência e certificação de que o procedimento está sendo realizado no paciente correto. Como homônimos podem gerar erros, é imprescindivel informar e registrar o nome completo na passagem de plantão(3).

O estado de saúde e a história prévia interferem diretamente nas condições clínicas e recuperação cirúrgica, sendo relevante estar ciente dessas informações. O sítio cirúrgico indica também maior atenção para transferir o paciente da maca para a cama, o posicionamento de sondas e drenos e os cuidados necessários ao pós-operatório na Terapia Intensiva. O sangramento e a utilização de drogas vasoativas no transoperatório podem indicar estados de choque, que deverão ser monitorados no POI, sendo essa informação importantíssima para quem recebe o paciente e continua seu cuidado na UTI.

Pesquisa revelou a necessidade de melhorias na passagem de plantão do CC para a UTI, haja vista que informações relativas à identificação dos pacientes, às intercorrências no intraoperatório, alergias prévias, estado clínico e os cuidados pós-operatórios recomendados não são repassadas para os profissionais da UTI(13)

A hemotransfusão também precisa ser comunicada, pois o enfermeiro intensivista deve atentar-se à ocorrência de reações transfusionais, que podem ter caráter imediato (até $24 \mathrm{~h}$ após a transfusão) ou tardio (mais de $24 \mathrm{~h})^{(14)}$. Outras questões referentes à utilização de catéteres, drenos e monitorizações de pressões invasivas devem ser informadas previamente pelo risco de coagulação do sistema e perdas do dispositivo. Informações do intraoperatório, como transfusão sanguínea, e aquelas relativas ao pós-operatório, a exemplo da utilização de drenos, sondas e curativos, foram consideradas na elaboração de um impresso para passagem de plantão, desenvolvido para melhorar a rotina de um hospital geral privado e filantrópico do municipio de São Paulo(5), informações também incorporadas no checklist proposto no presente estudo. 
Pesquisa sobre o transporte intra-hospitalar de pacientes críticos evidencia falha de comunicação entre equipes e falta de conhecimento do profissional como fatores relacionados aos eventos adversos. Assim, transportar paciente crítico de modo seguro implica melhorar a comunicação entre as equipes, bem como padronizar ações e equipamentos utilizados por meio de protocolos, destacando-se o papel fundamental do enfermeiro na difusão das informações ${ }^{(15)}$.

Como limitação do estudo, aponta-se a elaboração do checklist por profissionais de enfermagem de uma única instituição, pela possibilidade de considerarem somente a realidade institucional no desenvolvimento do instrumento, o que pode dificultar a sua implementação na passagem de plantão em outros contextos de cuidado intensivo. Contudo, sugere- se a implementação do checklist em diferentes instituições hospitalares, a fim de aperfeiçoar esta ferramenta, com vistas à segurança do paciente crítico.

\section{CONCLUSÃO}

A passagem de plantão é percebida como essencial ao planejamento do cuidado seguro ao paciente admitido na UTI proveniente do CC. O checklist elaborado contempla informações essenciais para conhecer a situação clinica do paciente imediatamente após a cirurgia e até sua chegada na UTI. O instrumento poderá contribuir para a continuidade de cuidados seguros no pós-operatório imediato e mediato na UTI, sendo a sua objetividade um facilitador à implementação pelos profissionais.

\section{REFERÊNCIAS}

1. Marques LF, Santiago LC, Felix VC. A passagem de plantão como elemento fundamental no processo de cuidar em enfermagem: o perfil da equipe de enfermagem de um hospital universitário. R. Pesq.: cuid. fundam. Online. 2012; 4(2) [acesso 01 mar 2016]. Disponivel: http://www.seer.unirio.br/index. php/cuidadofundamental/article/view/1567/pdf_500.

2. Agência Nacional de Vigilância Sanitária (ANVISA). Boletim Informativo: segurança do paciente e qualidade em serviços de saúde. 2011; 1(1) [acesso ol mar 2016]. Disponivel: http://portal.anvisa.gov.br/wps/wcm/connect/ f72c20804863ald88cc88d2bd5b3ccfO/BOLETIM+I.PDF?MOD=AJPERES

3. Mello JF, Barbosa SFF. Cultura de segurança do paciente em terapia intensiva: recomendações da enfermagem. Texto Contexto Enferm. 2013; 22(4):1124-33.

4. Minuzzi AP, Salum NC, Locks MOH, Amante LN, Matos E. Contribuições da equipe de saúde visando à promoção da segurança do paciente no cuidado intensivo. Escola Anna Nery. 2016; 20(1):121-9.

5. Sousa CS, Souza RCS, Gonçalves MC, Diniz TRZ, Cunha ALSM. Comunicação efetiva entre o centro cirúrgico e a unidade de terapia intensiva. Rev SOBECC. 2014; 19(1):44-50.

6. Sarabia C, Carmen M, Guerra G de la G, Manuel J, Campo GM, Manrique BT. El checklist: avance hacia la excelencia en calidad asistencial. Metas Enferm. 2013; 16(7). graf, tab.

7. Lefèvre F, Lefèvre AMC. Pesquisa de representação social: um enfoque qualiquantitativo: a metodologia do Discurso do Sujeito Coletivo. 2 ed. Brasilia: Liber Livro; 2012

8. Ong MS, Magrabil F, Post J, Morris S, Westbrook J, Wobcke W, et al. Communication interventions to improve adherence to infection control precautions: a randomised crossover trial. BMC Infectious Diseases. 2013 $13: 72$.

9. Bohrer CD, Marques LGS, Vasconcelos RO, Oliveira JLC, Nicola AL, Kawamoto AM. Comunicação e cultura de segurança do paciente no ambiente hospitalar: visão da equipe multiprofissional. Rev Enferm UFSM. 2016; 6(1):50-60.

10. El-Jardali F, Sheikh F, Garcia NA, Jamal D, Abdo A. Patient safety culture in a large teaching hospital in Riyadh: baseline assessment, comparative analysis and opportunities for improvement. BMC Health Services Research. 2014; 14:122.

11. Halm MA. Nursing handoffs: ensuring safe passage for patients. American Journal of Critical Care. 2013; 22(2):158-62.

12. Chenault K, Moga Michael-Alice, Shin M, Petersen E, Backer C, Oliveira Jr GS, Suresh S. Sustainability of protocolized handover of pediatric cardiac surgery patients to the intensive care unit. Pediatric Anesthesia. 2016; 26(5):488-94

13. Bueno BRM, Moraes SS, Suzuki K, Gonçalves FAF, Barreto RASS, Gebrim CFL. Caracterização da passagem de plantão entre o centro cirúrgico e a unidade de terapia intensiva. Cogitare Enferm. 2015; 20(3):512-8.

14. Barbosa SM, Torres CA, Gubert FA, Pinheiro PNC, Vieira NFC Enfermagem e a prática hemoterápica no Brasil: revisão integrativa. Acta Paul Enferm. 2011; 24(1):132-6.

15. Almeida ACG, Neves ALD, Souza CLB, Garcia JH, Lopes JL, Barros ALBL. Transporte intra-hospitalar de pacientes adultos em estado crítico: complicações relacionadas à equipe, equipamentos e fatores fisiológicos. Acta Paul Enferm. 2012; 25 (3):471-6. 